\title{
Observation on the Application of "Online + Offline" Mixed Teaching Mode in Clinical Nursing Teaching
}

\author{
Guo Tang\#*, Hui Sun\# \\ First Medical Center of PLA General Hospital Pain Department, Beijing 100853, China \\ \# These authors contributed equally to this work \\ *Corresponding author: Guo Tang, 1838716933@qq.com
}

Copyright: (C) 2022 Author(s). This is an open-access article distributed under the terms of the Creative Commons Attribution License (CC BY 4.0), permitting distribution and reproduction in any medium, provided the original work is cited.

\begin{abstract}
Objective: To observe the application effect of "online + offline" mixed teaching mode in clinical nursing teaching. Methods: A total of 72 clinical nursing workers, who work in PLA General Hospital and participated in the experiment from March 2019 to March 2021, were selected as the subjects of this study. According to the odd numbers assigned to the participants' names, the participants were divided into two groups: the experimental group and the control group, with 36 cases in each group. The experimental group received "online + offline" hybrid teaching, whereas the control group received traditional offline teaching. In order to evaluate the efficacy and safety of the two groups of nursing workers, their assessment scores, teaching satisfaction scores, and teaching evaluation indicators were observed. Results: The experimental group was significantly better than the control group in terms of assessment scores, teaching satisfaction scores, and teaching evaluation indicators $(P<0.05)$. Conclusion: In clinical nursing teaching, the "online + offline" mixed teaching mode plays a unique role in improving students' scores and teaching quality; thus, it should be promoted.
\end{abstract}

Keywords: Mixed teaching mode; Assessment results; Clinical nursing teaching

Online publication: February 22, 2022

\section{Introduction}

The traditional learning mode of clinical nursing workers is offline teaching. The situation where one teacher is responsible for a number of students results in a phenomenon where it is impossible to meet the learning demand of each and every student. At the same time, the burden of teachers is becoming heavier, especially in the current environment. At present, the national interest is for medical-related content; hence, improving the quality of teaching and the skills of fresh blood is the key issue ${ }^{[1,2]}$. The "online + offline" mixed teaching mode enables students to use fragmented time to learn; it also provides online and offline learning sites, which is an ice-breaking development in clinical teaching ${ }^{[3]}$. This study examines the application effect of "online + offline" mixed teaching mode in clinical nursing teaching.

\section{Data and methods}

\subsection{Clinical data}

A total of 72 clinical nursing workers were selected from PLA General Hospital from March 2019 to March 2021 to participate in the study. There were 21 male workers and 41 female workers. Their age ranged from 18 to 39 years old, with an average age of $22.0 \pm 6.0$ years. All clinical nursing workers, who met the 
inclusion criteria, had signed the informed consent prior to the approval of their medical superior. The study excluded those who had quit midway and those with improper learning attitudes. The participants were divided into two groups according to their names and strokes; odd numbers were grouped in the experimental group and even numbers were grouped in the control group, with 36 cases in each group. In the experimental group, there were 16 male workers and 20 female workers, age ranging from 18 to 39 years old, with an average age of $22.0 \pm 6.0$ years. The age range of the control group was 18 to 39 years old, with an average of $22.0 \pm 6.0$ years. There was no statistical significance $(P>0.05)$ in the clinical data between the two groups, indicating a certain comparability.

\subsection{Inclusion and exclusion criteria}

The study included those who actively cooperate. The study excluded those who quit the study midway and those with poor learning attitude ${ }^{[4,5]}$.

\subsection{Methods}

For the control group, conventional offline teaching was carried out by instructors according to hospital regulations and their previous teaching experience. Theoretical teaching was mainly carried out via explanation by the instructors and questions from the students. Clinical operation was carried out via onsite operation and explanation by the instructors in addition to regular offline assessment.

For the experimental group, "online + offline" mixed teaching was carried out on the basis of the control group. The teaching mode includes the enterprise WeChat development platform, industry-related information content, health reports, schedules, and meetings. Online teaching was mainly carried out via live and micro videos in a micro dynamic manner. Test contents, online assessments, and assessment contents were implemented consistently.

\subsection{Effect judgment criteria}

\subsubsection{Assessment results}

The assessment method or standard is as follows: the assessment was organized by the two groups at the same time, with the same content. Outstanding results indicate above excellent, effective results indicate above pass, and ineffective results indicate fail ${ }^{[6]}$.

\subsubsection{Teaching satisfaction}

The evaluation method or standard is as follows: the participants graded their teaching satisfaction degree with a total score of 100 . The higher the score, the higher the degree of satisfaction in teaching ${ }^{[7]}$.

\subsubsection{Teaching evaluation}

The evaluation method or standard is as follows: after teaching, the teachers evaluated the participants' theoretical and operational satisfaction. High satisfaction reflects an obvious effect, medium satisfaction for effective effect, and low satisfaction for ineffective effect.

\subsection{Statistical analysis}

Data were analyzed using SPSS 22.0. Measurement data and count data (\%) were used, and T test and chisquare $\left(\mathrm{X}^{2}\right)$ test were carried out. $P<0.05$ was considered statistically significant.

\section{Results}

\subsection{Comparing the assessment results between the two groups}

The assessment result of the experimental group was significantly better than that of the control group $(P$ 
$<0.05)$, as shown in Table 1.

Table 1. Comparison of assessment results between the two groups $(\overline{\mathrm{x}} \pm \mathrm{s})$

\begin{tabular}{cccc}
\hline Group & $\mathrm{n}$ & Theoretical results & Operating performance \\
\hline Experimental group & 36 & $93.32 \pm 4.27$ & $96.25 \pm 1.21$ \\
Control group & 36 & $81.62 \pm 5.29$ & $89.19 \pm 2.75$ \\
$t$ & & 116.32 & 131.28 \\
$P$ & & $<0.05$ & $<0.05$ \\
\hline
\end{tabular}

\subsection{Comparing the teaching satisfaction scores between the two groups}

The teaching satisfaction score of the experimental group was significantly better than that of the control group $(P<0.05)$, as shown in Table 2.

Table 2. Comparison of teaching satisfaction scores between the two groups $(\overline{\mathrm{x}} \pm \mathrm{s})$

\begin{tabular}{ccc}
\hline Group & $\mathrm{n}$ & Teaching satisfaction score \\
\hline Control group & 36 & $72.6 \pm 3.4$ \\
Experimental group & 36 & $92.6 \pm 2.5$ \\
$t$ & & 42.323 \\
$P$ & & 0.000 \\
\hline
\end{tabular}

\subsection{Comparing the teaching evaluation indicators between the two groups}

The teaching evaluation indicators of the experimental group were significantly higher than that of the control group $(P<0.05)$, as shown in Table 3 .

Table 3. Comparison of teaching evaluation indicators between the two groups (n/\%)

\begin{tabular}{ccccccc}
\hline \multirow{2}{*}{ Project } & \multicolumn{2}{c}{ Experimental group $(\mathrm{n}=36)$} & \multicolumn{2}{c}{ Control group $(\mathrm{n}=36)$} & \multirow{2}{*}{$x^{2}$} & \multirow{2}{*}{$P$} \\
\cline { 2 - 5 } & Satisfied & Dissatisfied & Satisfied & Dissatisfied & & \\
\hline Theoretical & 25 & 11 & 14 & 22 & 7.351 & $<0.05$ \\
Operational & 26 & 10 & 17 & 19 & 7.264 & $<0.05$ \\
\hline
\end{tabular}

\section{Discussion}

At present, social and economic development is becoming more rapid, people are becoming more health conscious, and more emphasis is on medical-related matters. The professional level of clinical nursing staffs is directly related to the staffs themselves, and it influences people's judgment on the hospital. This indicates that the theoretical basis and operation skills of clinical nursing staffs are important links. At present, the education mode for clinical nursing staffs is mainly offline teaching.

"Online + offline" mixed teaching mode conforms to the requirements of social development and improves the teaching quality. This model does not take up the offline teaching time but guarantees that offline teaching is carried out steadily. It provides another development platform for both students and teachers. WeChat is assumed as the carrier, and various software are used as teaching tools. In addition to reducing the operation difficulty, it is more convenient for students to use fragmented time for learning, and they can be assessed synchronously in various environments, thus reducing site requirements, which is convenient for teachers, students, and patients. 
Through the observation of these 72 clinical nursing workers, the assessment scores, teaching satisfaction scores, and teaching evaluation indicators of the experimental group ("online + offline" mixed teaching mode) were significantly better than those of the control group $(P<0.05)$.

In conclusion, it can be seen that the application of "online + offline" mixed teaching mode has outstanding effects in clinical nursing teaching; thus, it is worthy of clinical reference.

\section{Disclosure statement}

The authors declare no conflict of interest.

\section{References}

[1] Li C, Xiang J, Tan Y, 2020, Application of Hybrid Teaching Mode Based on WeChat Platform in Clinical Nursing Skills Teaching. Nursing Research, 656(12): 157-160.

[2] Mei H, Hu M, Huang L, et al., 2019, Application of Online and Offline Teaching Methods in Clinical Anesthesiology Teaching. Chinese Journal of Medical Education, 39(11): 843-846.

[3] Feng H, Liu F, Li C, et al., 2020, Preliminary Study on Online and Offline Blended Teaching of Medical Immunology in " $5+3$ " Integrated Undergraduate Teaching. Chinese Journal of Immunology, 8(18): 982.

[4] Fu B, Du X, He M, 2019, Practical Research on the Blended Teaching of Nursing Etiquette Based on MOOCs. Nursing Research, 636(16): 159-161.

[5] Zhang Y, Li S, Xu M, et al., 2019, The Application of Blended Teaching in the Cultivation of Humanistic Care Ability of New Nurses. Shanghai Nursing, 19(3): 71-73.

[6] Qu K, 2020, Numerical Analysis Teaching Method and Research based on Online and Offline Hybrid Teaching Model. Education Research, 3(3): 118-119.

[7] Geng J, 2020, Online and Offline Mixed Teaching of Ideological and Political Education in Higher Vocational Education Under the Internet Environment. Food Research and Development, 97(24): 279.

\section{Publisher's note}

Bio-Byword Scientific Publishing remains neutral with regard to jurisdictional claims in published maps and institutional affiliations. 